\title{
Quand les bactéries nous montrent comment fonctionnent les récepteurs humains
}

Stefano Marullo Brigitte Bertin Michael Freissmuth A. Donny Strosberg

\section{ADRESSES}

S. Marullo : docteur en médecine, docteur ès sciences, chargé de recherche à l'Inserm.

B. Bertin : docteur is sciences, chercheur post-doctoral.

A. D. Strosberg : professeur, directeur du laboratoire. Laboratoire d'immunopharmacologie moléculaire UPR 415 Cnrs, université ParisVII, ICGM, 22, rue Méchain, 75014 Paris France.

M. Freissmuth : professeur assistant. Institut de pharmacologie, université de Vienne, Währinger Strasse, 13a A1090 Vienne, Autriche.

Les récepteurs membranaires couplés aux protéines $\mathbf{G}$ occupent une place prépondérante dans la régulation d'un grand nombre de fonctions vitales de l'organisme. Les protéines $\mathbf{G}$ comprennent trois sous-unités appelées $\alpha, \beta$ et $\gamma$. La sous-unité $\alpha$ est responsable du couplage avec les récepteurs et de l'activation des effecteurs cellulaires (enzymes, canaux ioniques). Il a été montré que les récepteurs de cette famille, exprimés dans Escherichia coli, peuvent conserver intactes leurs propriétés de liaison des ligands. Les sous-unités $\alpha$ de plusieurs protéines $\mathbf{G}$ ont également été exprimées dans les bactéries, et, après purification, elles sont capables d'interagir avec des récepteurs. Ces résultats ont conduit à mettre au point un procédé de reconstitution in vitro du couplage entre récepteurs et protéines $\mathbf{G}$ recombinants, exprimés dans Escherichia coli. Par cette approche, il est possible de mesurer des préférences de couplage entre un récepteur donné et plusieurs protéines $G$ différentes sans risque d'interférence avec des récepteurs ou des protéines $G$ endogènes.

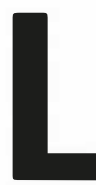

'expérience de notre laboratoire dans le domaine de l'expression dans $E$. coli des récepteurs membranaires couplés aux protéines $G$ transductrices (ainsi appelées parce qu'elles ont la propriété de lier et d'hydrolyser le GTP au cours de leur état d'activation) remonte à presque cinq ans. A cette époque, le gène d'un des premiers récepteurs humains appartenant à cette vaste famille, le récepteur $\beta 2$-adrénergique, venait d'être cloné dans le laboratoire.

Des récepteurs humains exprimés dans les bactéries conservent intactes leurs propriétés de liaison

Afin de produire des anticorps spécifiques de ce récepteur, une protéine 


\section{RÉFÉRENCES}

1. Henderson R, Unwin PNT. Threedimensional model of purple membrane obtained by electron microscopy. Nature 1975 ; 257 : 28-32

2. Dixon RAF, Kobilka BK, Strader DJ, et al. Cloning of the gene and cDNA for mammalian $\beta$-adrenergic receptor and homology with rhodopsin. Nature 1986 ; 321: 75-9.

3. Dunn RJ, Hackett NR, McCoy JM, Chao BH, Kimura K, Korana GH. Structure-function studies on bacteriorhodopsin. I. Expression of the bacterioopsin gene in Escherichia coli. J Biol Chem 1987 ; 262 9246-54.

4. Marullo S, Delavier-Klutchko C, Eshdat Y, Strosberg AD, Emorine IJJ. Human $\beta$-2-adrenergic receptors expressed in Escherichia coli membranes retain their pharmacological properties. Proc Natl Acad Sci USA $1988 ; 84: 7551-5$.

5. Chapot MP, Eshdat Y, Marullo S, et al. Localization and characterization of three different vertebrate $\beta$-adrenergic receptors expressed in Escherichia coli. Eur $J$ Biochem $1990 ; 187: 137-44$.

6. Marullo S, Delavier-Klutchko C, Guillet JG, Charbit A, Strosberg AD, Emorine LJ. Expression of human $\beta 1$ and $\beta 2$ adrenergic receptors in $E$. coli as a new tool for ligand screening. Biotechnology $1989 ; 7$ : 923-7.

7. Bertin B, Freissmuth $M$, Breyer $R$ Schütz W, Strosberg AD, Marullo S. Functional expression of the human serotonin 5-HT1A receptor in Escherichia coli : ligand binding properties and interaction with recombinant $\mathrm{G} \alpha$ proteins. J Biol Chem 1992 (sous presse).

8. Birnbaumer L, Abramowitz J, Yatani A, et al. Roles of $\mathrm{G}$ proteins in coupling of receptors to ionic channels and other effector systems. Crit Rev Biochem Mol Biol 1990 ; $25: 225-44$.

9. Gilman AG. G proteins and dual control of adenylate cyclase. Cell 1984; 36 577-9

10. Simon MI, Strathmann MP, Gautam N. Diversity of $G$ protein in signal transduction. Science 1991; 252 : 802-8. de fusion entre la région codante du récepteur $\beta 2$ humain et la $\beta$ galactosidase a été construite dans le phage $\lambda-G T 11$. Une fois les clones recombinants obtenus, nous avons recherché une activité de liaison pour un ligand $\beta 2$-adrénergique radiomarqué (l'iodocyanopindolol) dans le lysat des bactéries infectées par le phage. En effet, la bactériorhodopsine, une pompe à protons de Halobacterium halobium, comportant sept hélices transmembranaires [1], et qui est encore actuellement le modèle de structure de tous les récepteurs couplés aux protéines $\mathrm{G}$ [2], avait pu être exprimée dans $E$. coli sous une

forme active [3]. La surprise n'a donc pas été d'observer l'activité de liaison recherchée, mais de constater que, dans les bactéries, les récepteurs $\beta 2$-adrénergiques humains conservaient toutes leurs propriétés de liaison : saturabilité, niveau d'affinité pour les ligands $\beta$-adrénergiques, stéréosélectivité et spécificité pour les ligands sélectifs du sous-type $\beta 2$ [4]. Ces résultats suggéraient que le site de liaison du ligand était fidèlement reconstitué dans la membrane bactérienne et confirmaient que la nonglycosylation des récepteurs $\beta$ n'affecte pas l'intégrité du site de liaison [4], dans la mesure où les pro-

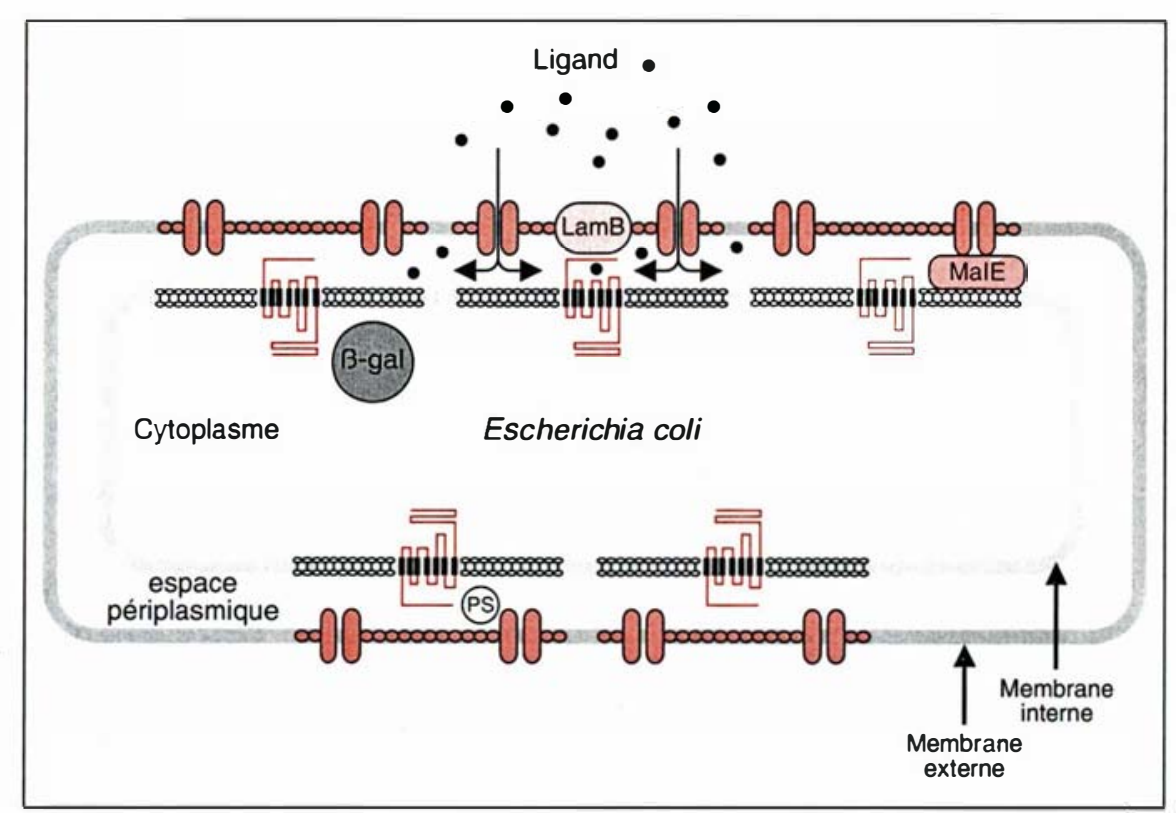

Figure 1. Schéma illustrant les différents procédés utilisés pour l'expression des récepteurs $\beta$-adrénergiques dans E.coli. Dans le premier système $d^{\prime}$ expression, la région codante des récepteurs $\beta$-adrénergiques a été fusionnée en $3^{\prime}$ du gène de la $\beta$-galactosidase qui est une protéine cytoplasmique. $D$ 'autres types de fusion génique ont été réalisés successivement avec les gènes bactériens LamB et MalE, codant pour des protéines impliquées dans le transport du maltose, qui se localisent respectivement dans la membrane externe et l'espace périplasmique de $\mathrm{E}$. coli. Dans les trois cas, les récepteurs capables de lier les ligands sont spontanément clivés de leur partenaire de fusion et ne constituent qu'une faible fraction de la totalité des protéines de fusion effectivement synthétisées. Par marquage de photo-affinité et séparation des membranes bactériennes, il a été montré que ces formes " actives" sont principalement localisées au niveau de la membrane interne, dont la structure est proche de celle des membranes cytoplasmiques des organismes supérieurs. Cela a également été observé avec les deux autres procédés d'expression, dans lesquels la région codante des récepteurs a été insérée à une position située à une soixantaine de bases après le peptide-signal de MalE IPS dans le schémal ou directement sous le contrôle du promoteur T7. Dans les expériences de liaison sur cellules intactes, les ligands diffusent jusqu'aux récepteurs en franchissant la membrane externe à travers des pores suffisamment grands pour que les constantes de liaison ne soient pas affectées. 
téines exprimées dans $E$. coli ne sont pas glycosylées. Par des expériences de marquage d'affinité et d'immunochimie sur membranes fractionnées [5], il a été montré que les récepteurs actifs (en termes de liaison des ligands) se localisent au niveau de la membrane interne des bactéries dont la structure phospholipidique est proche de celle des membranes cytoplasmiques des organismes supérieurs. Enfin, le poids moléculaire des récepteurs actifs dans les membranes bactériennes indique que le partenaire de fusion est clivé spontanément.

Depuis ce premier succès, les rendements d'expression du récepteur $\beta 2$-adrénergique ont été améliorés grâce à l'utilisation de nouveaux vecteurs (figure 1) [6] et d'autres gènes codant pour des récepteurs de cette famille, comme le récepteur $\beta 1$-adrénergique [6] et le récepteur sérotoninergique 5-HT1A [7] ont pu être exprimés dans $E$. coli avec des résultats équivalents en ce qui concerne la qualité de reconstitution des propriétés de liaison.

\section{Certitudes et incertitudes concernant les protéines $\boldsymbol{G}$ transductrices}

Jusqu'à présent, au moins 85 récepteurs couplés aux protéines $G$ trimériques (par opposition aux petites protéines $G$ de la famille de l'oncogène ras) ont été identifiés par clonage des gènes ou par caractérisation biochimique et pharmacologique [8]. Ces protéines $G$ comprennent trois sous-unités appelées $\alpha, \beta$ et $\gamma$. De nombreux travaux, en particulier ceux concernant le système de signalisation récepteur $\beta$-adrénergique protéine Gs - adénylate cyclase, ont permis de comprendre le mécanisme d'activation de ces molécules transductrices [9] (figure 2). La sous-unité $\alpha$ se caractérise par un site d'interaction avec le récepteur, un site de liaison de haute affinité pour les nucléotides guanyliques et une activité GTPase intrinsèque. A l'état basal (en l'absence d'activation par le récepteur), elle lie le GDP avec une haute affinité. Après liaison de l'agoniste, le récepteur activé interagit avec la protéine $G$ appropriée et accélère le relargage de GDP. Dans le complexe ternaire agoniste$\mathrm{m} / \mathrm{s} n^{\circ} 6$, vol. 8, juin-juillet 92

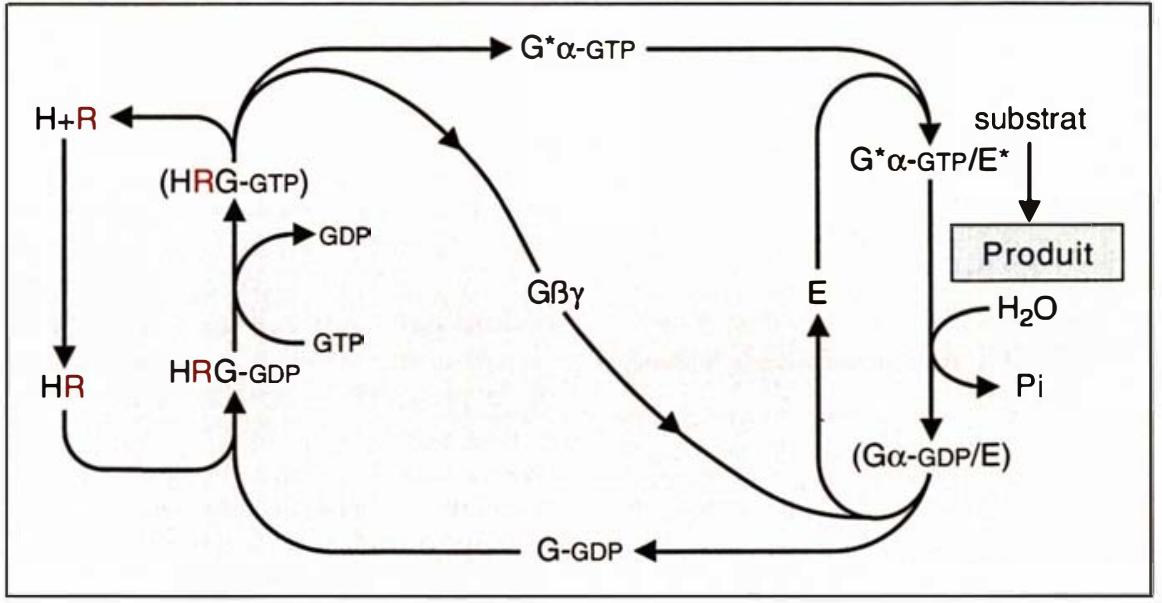

Figure 2. Schéma illustrant : (1) l'activation de la protéine $G$ par le récepteur en présence d'un ligand agoniste, (2) la stimulation de l'effecteur par la protéine $G$ activée, (3) la relaxation du système d'activation, avec retour à l'état basal. Les détails de ce schéma sont exposés dans le texte. $H$ : hormone ; $R$ : récepteur; $G$ : protéine $G$ trimérique; $G \alpha^{*}$ et $G \beta \gamma$ : sousunités $\alpha$ activée et $\beta \gamma$ de la protéine $G ; E$ et $E^{*}$ : effecteurs à l'état basal et activé. Les complexes entre parenthèses sont éphémères. (D'après [21].)

récepteur-protéine $G$ ainsi formé, l'agoniste a une affinité pour le récepteur plus grande que pour le récepteur seul. Il est possible d'identifier ce complexe ternaire dans des préparations membranaires sous la forme de sites de liaison de haute affinité. Dans les cellules intactes, au contraire, le complexe ternaire est éphémère car, en présence des hautes concentrations de GTP intracellulaires, ce dernier se fixe quasi instantanément sur la sous-unité $\alpha$. En présence d'ions $\mathrm{Mg}^{2+}$, cette réaction aboutit à la formation de la sous-unité $\alpha$ activée, qui se dissocie du récepteur et des sous-unités $\beta$ et $\gamma$. La sous-unité $\alpha$ activée module l'activité de l'effecteur correspondant, par exemple l'adénylate cyclase. Au bout d'un certain temps, l'activité GTPase intrinsèque de la sous-unité $\alpha$ clive le troisième phosphate du GTP; la sousunité $\alpha$ est désactivée et se réassocie aux sous-unitéc $\beta \gamma$, remettant le système à l'état basal.

Par clonage des gènes ou par purification protéique, une vingtaine de sous-unités $\alpha$ ont été identifiées, que l'on regroupe sur la base de leur structure et de leur fonction en six familles : $G_{\mathrm{S}}, G_{\mathrm{T}}, \mathrm{G}_{\mathrm{J}}, \mathrm{G}_{\mathrm{O}}, \mathrm{G}_{\mathrm{Z}}$ et $\mathrm{G}_{\mathrm{Q}}[10]$. Les sous-unités $\alpha$ activent un ou plusieurs effecteurs et peuvent à leur tour être activées par plusieurs récepteurs différents ; certaines protéines $G$ récemment identifiées sont encore en quête de leur récepteur ou de leur effecteur. Il est de plus établi qu'un même récepteur peut activer éventuellement plusieurs sousunités $\alpha$ (souvent avec des affinités différentes) et donc être couplé à différents effecteurs. Quatre membres de chaque sous-unité $\beta$ et $\gamma$ ont été identifiés. Des résultats récents suggèrent qu'il y aurait des associations préférentielles entre une sous-unité $\alpha$ donnée et diverses combinaisons de $\beta$ et $\gamma$ [11]. De ces différentes constatations il apparaît que, comme le code génétique, le code de la signalisation est dégénéré, mais avec un plus grand nombre de combinaisons possibles.

Une étape supplémentaire dans la complexité, déjà grande, des voies de signalisation cellulaire est rapidement franchie si l'on prend en compte les nombreuses régulations qui les affectent. Des régulations croisées, inhibitrices ou activatrices, entre différentes voies de signalisation passant par les protéines $G$ ont en effet été démontrées: le découplage entre récepteurs $\beta$-adrénergiques et protéine $\mathrm{Gs}$ induit par la protéine kinase $\mathrm{C}$ $(\mathrm{PKC})$, suite à l'activation de la voie 


\section{RÉFÉRENCES}

11. Fawzi AB, Fay DS, Murphy EA, Tamir H, Erdos JJ, Northup JK. Rhodopsin and retinal $\mathrm{G}$-protein distinguish among $\mathrm{G}$-protein $\beta \gamma$ subunit forms. J Biol Chem 1991 ; 266 : 12194-200.

12. Hadcock JR, Ros M, Watkins DC, Malbon CC. Cross regulation between Gprotein mediated pathways. Stimulation of adenilyl cyclase increases expression of the inhibitory G-protein Gia-2. I Biol Chem 1990 ; 265 : 14784-90.

13. Graziano MP, Casey PJ, Gilman AG. Expression of cDNAs for $\mathrm{G}$ proteins in Escherichia coli. J Biol Chem 1987; 262 : 11375-81.

14. Graziano MP, Freissmuth M, Gilman AG. Expression of Gs $\alpha$ in Escherichia coli : purification and properties of two forms of the protein. J Biol Chem 1989 ; 264 ; 409-18.

15. Freissmuth $M$, Schütz $W$, Linder $M E$. Interactions of the bovine brain Al-adenosine receptor with recombinant $G$ protein $\alpha$-subunits. $J$ Biol Chem 1991 ; 266 : 17778-83.

16. Freissmuth $M$, Selzer E, Marullo S, Schütz W, Strosberg AD. Expression of two human $\beta$-adrenergic receptors in Escherichia coli : functional interaction with two forms of the stimulatory G protein. Proc Natl Acad Sci USA 1991; 88 : 85458-52.

17. Luyten WHML, Pauwels PJ, Moereels H, Marullo S, Strosberg AD, Leysen JE. Comparative study of the binding properties of cloned human $\beta 1$ and $\beta 2$-adrenergic receptors expressed in Escherichia coli. Drug Invest $1991 ; 3$ (suppl 1) : 3-12.

18. Strosberg AD, Marullo S. Functional expression of $\mathrm{G}$-protein coupled receptors in microorganisms. Trends Pharmacol Sci 1992 (sous presse).

19. Marullo S, Emorine L, Strosberg AD, Delavier Klutchko C. Selective binding of ligands to $\beta 1, \quad \beta 2$ or chimeric $\beta 1 / \beta 2$-adrenergic receptors involves multiple subsites. EMBO J 1990 ; 9 : 1471-6.

20. Breyer RM, Strosberg AD, Guillet JG. Mutational analysis of ligand binding activity of $\beta 2$ adrenergic receptor expressed in Escherichia coli. EMBO J 1990 ; 9 : 2679-84.

21. Freissmuth M, Casey PJ, Gilman A.G. $G$ proteins control diverse pathways of transmembrane signaling. FASEB $J 1989 ; 3$ : de dégradation du phosphatidyl inositol, en est un exemple. Les régulations croisées ne se limitent pas à des interactions entre protéines, mais affectent également la synthèse des protéines qui interviennent dans les voies de signalisation. Ainsi, il a été montré que la stimulation d'adénylate cyclase par l'activation de la protéine $\mathrm{G}$ stimulatrice augmente l'expression de la protéine $G$ inhibitrice $G_{i \alpha 2}$ [12] L'idée qui émerge de ces données est que la signalisation cellulaire ne peut plus être considérée comme une simple superposition de voies d'activation indépendantes, mais plutôt comme un réseau de signaux interactifs. L'élucidation de tous les éléments de ce réseau, et le tri entre couplages effectivement possibles et couplages non fonctionnels, permettra de mieux comprendre les bases moléculaires de l'activation cellulaire et d'identifier éventuellement de nouvelles cibles pour la modulation pharmacologique de la signalisation.

\section{Étude de couplage entre récepteurs et protéines $\boldsymbol{G}$}

$\mathrm{Si}$ certaines voies d'activation par les récepteurs sont parfaitement connues dans leur détails, d'autres le sont beaucoup moins. Plusieurs méthodes ont été utilisées pour définir à quelles sous-unités $\alpha$ un récepteur donné peut se coupler; en voici quelques exemples.

- Certaines toxines bactériennes comme celle produite par Bordetella pertussis ont la propriété de bloquer l'interaction entre récepteur et certaines protéines $\mathrm{G}$ en induisant l'ADP. ribosylation de ces demières au niveau de la région d'interaction avec le récepteur. L'utilisation de cette toxine sert à identifier les voies de signalisation passant par l'activation des protéines des groupes $\mathrm{Gi}$ et $\mathrm{Go}$, mais ne permet pas de discriminer entre les différents sous-types à l'intérieur de ces groupes.

- La combinaison du marquage par affinité des protéines $\mathrm{G}$ activées par un dérivé azido du GTP, avec la migration en gel dénaturant, et l'immunoempreinte avec des anticorps spécifiques des différentes sous-unités $\alpha$ est une technique plus récente. Suite à une stimulation hormonale donnée, seule la (ou les) protéine(s) G acti- vée(s) par le récepteur peu(ven)t échanger le GDP avec le dérivé azido radiomarqué du GTP, qui se lie de façon irréversible. Les limites de cette méthode tiennent à la spécificité des anticorps (les séquences des sousunités $\alpha$ sont très homologues), au pouvoir résolutif insuffisant des gels et au fait que toutes les sous-unités $\alpha$ ne sont probablement pas encore identifiées.

- Les expériences de reconstitution in vitro dans des vésicules phospholipidiques à partir de protéines purifiées des tissus sont expérimentalement très lourdes. De plus, même une purification très poussée ne permet pas d'éliminer une contamination éventuelle par une protéine $\mathrm{G}$ encore inconnue. - La micro-injection d'ARN antisens pour bloquer sélectivement la synthèse d'une sous-unité $\alpha$ est une méthode efficace, mais elle est réservée à des voies d'activation mesurables au niveau d'une seule cellule (en patchclamp, par exemple).

Le fait que les récepteurs $\beta$-adrénergiques recombinants exprimés dans $E$. coli conservent toutes leurs propriétés de liaison nous suggérait que les caractéristiques physicochimiques des membranes bactériennes pouvaient également permettre la reconstitution d'une conformation fonctionnelle des récepteurs. Plusieurs ADNc codant pour les sous-unités $\alpha$ des protéines $\mathrm{G}$ avaient déjà été exprimés dans les bactéries par l'équipe de Gilman. Bien que les différentes modifications post- ou cotraductionnelles des sous-unités $G \alpha$ produites dans les cellules de mammifère ne se réalisent pas dans $E$. coli, ces protéines recombinantes conservent, au moins en partie, leurs propriétés fonctionnelles [13, 14]. Nous avons ainsi été amenés à étudier la possibilité de reconstituer le couplage entre récepteurs $\beta$-adrénergiques et sous-unités $\alpha$ s recombinants exprimés dans les bactéries.

\section{Couplage fonctionnel entre récepteurs $\beta 2$-adrénergiques et protéines Gs $\alpha$ exprimés dans E. coli}

L'intégrité fonctionnelle des récepteurs $\beta 1$ et $\beta 2$-adrénergiques exprimés dans $E$. coli a été étudiée en analysant le couplage du récepteur avec la 
sous-unité Gos, également exprimée dans les bactéries. Le procédé de reconstitution que nous avons utilisé peut se résumer comme suit (figure 3). Les récepteurs et les sous-unités $\alpha$ sont exprimés dans des bactéries différentes. Les membranes des bactéries contenant les récepteurs sont préparées par digestion avec le lysozyme, choc osmotique et centrifugation. Les sous-unités $\alpha$ recombinantes (que nous désignerons dorénavant par la lettre r) sont purifiées à partir des bactéries et solubilisées par des détergents. Les sous-unités $\beta \gamma$ sont purifiées à partir de cerveau bovin [15] car, jusqu'à présent, il n'a pas été possible de les exprimer dans les bactéries en maintenant leur conformation active. Ces différentes protéines sont réunies pour reconstituer le couplage. Nous avons vu plus haut que le couplage entre récepteurs et protéines $G$ se traduit par l'augmentation d'affinité du récepteur pour l'agoniste, puis par l'échange GDPGTP au niveau du site de liaison pour les nucléotides guanyliques de la sous-unité $\alpha$. Ces deux paramètres ont été étudiés pour caractériser la reconstitution du couplage fonctionnel entre récepteurs et protéines G recombinants dans $E$. coli $[16]$.

Les expériences de compétition de liaison d'un antagoniste radiomarqué par l'isoprotérénol (agoniste de référence des récepteurs $\beta$-adrénergiques) montrent que l'adjonction de rGs $\alpha$ $\beta \gamma$ solubilisées reconstitue effectivement, au niveau du récepteur, des sites de liaison de haute affinité pour l'agoniste. Ces sites disparaissent en présence de [GTP $\gamma$-S], un analogue non hydrolysable du GTP qui dissocie le complexe ternaire.

Les mêmes expériences de reconstitution ont été faites avec rGs $\alpha$-PT, une sous-unité $\alpha$ s mutée près de l'extrémité carboxyterminale (aspartique ${ }^{390}$-Cystéine ${ }^{391}$-Glycine ${ }^{392}$ au lieu de glutamine-tyrosine-acide glutamique) connue pour son mauvais couplage avec les récepteurs $\beta$, et une sous-unité inhibitrice de l'adénylate cyclase rGi $\alpha-1$. Ces deux sous-unités sont, respectivement, 20 fois et 200 fois moins actives que deux variantes d'épissage ( $\mathrm{rGs} \alpha-\mathrm{S}$ et $\mathrm{rGs} \alpha-\mathrm{L}$ ) de la sous-unité Gs $\alpha$, pour la reconstitution des sites de haute affinité. Cela indique que la spécificité d'interaction qui existe dans les cellules de mam-

Figure 3. Mise en évidence du couplage entre récepteurs $\beta 2$-adrénergiques et sous-unités $\alpha$ recombinantes des protéines $\mathbf{G}$ exprimés dans $\mathbf{E}$. coli. Les récepteurs et les sous-unités $\alpha$ des protéines $G$ sont exprimés séparément dans $\mathrm{E}$. coli. Pour obtenir la reconstitution, les sous-unités $\alpha$, solubilisées par des détergents et purifiées à partir des bactéries, et les sousunités $\beta$ et $\gamma$, purifiées à partir de cerveau bovin, sont mises en présence de membranes bactériennes contenant les récepteurs. La réalisation du couplage fonctionnel entre ces différentes molécules est évaluée par deux paramètres : (1) apparition dans les membranes bactériennes de sites récepteurs de haute affinité pour les ligands agonistes; (2) stimulation par les ligands agonistes de l'incorporation d'un analogue non hydrolysable du GTP par la protéine G, à travers l'activation des récepteurs. (a) En présence de la sous-unité Gs $\alpha$ recombinante et des sous-unités $\beta \gamma$ exogènes, deux sites de liaison de haute et basse affinité pour l'isoprotérénol sont observés dans les membranes bactériennes exprimant le récepteur $\beta 2$-adrénergique. L'addition d'un analogue non hydrolysable du GTP (GTP $\gamma$ S) qui dissocie le complexe ternaire H-R-G fait disparaître les sites de haute affinité. (b) Titration du récepteur $\beta 2$-adrénergique en présence de concentrations croissantes de sous-unités $G \alpha$ recombinantes. Dans le milieu réactionnel, les récepteurs $\beta$ sont en présence de concentrations fixes de ligand antagoniste radioactif (le ICYP) et d'agoniste (I'isoprotérénol). Plus on ajoute de sous-unité $G \alpha$, plus la proportion de sites à haute affinité augmente et plus le ligand radioactif est déplacé par l'isoprotérénol. II est possible de comparer par cette approche, l'affinité apparente du couplage entre les récepteurs et différentes sous-unités $\alpha$, comme, ici, la forme d'épissage longue de la Gs $\alpha(G s \alpha-L)$, une forme mutée moins active de celleci $(G s \alpha-P T)$ et la protéine Gio-1, inhibitrice de l'adénylate cyclase. (c) Courbe d'incorporation du GTP $S$ radiomarqué dans la sous-unité Gs $\alpha$ en fonction du temps et en présence des membranes bactériennes exprimant des récepteurs $\beta 2$-adrénergiques. L'isoprotérénol (Iso) accélère l'incorporation, alors que le propranolol, un antagoniste $\beta$-adrénergique, la ralentit.

mifère est également retrouvée dans ce système de reconstitution.

Après incorporation stable de $\mathrm{rGs} \alpha$ $\beta \gamma$ dans les membranes bactériennes exprimant le récepteur $\beta 2$, l'activation de la protéine Gs par le récep-
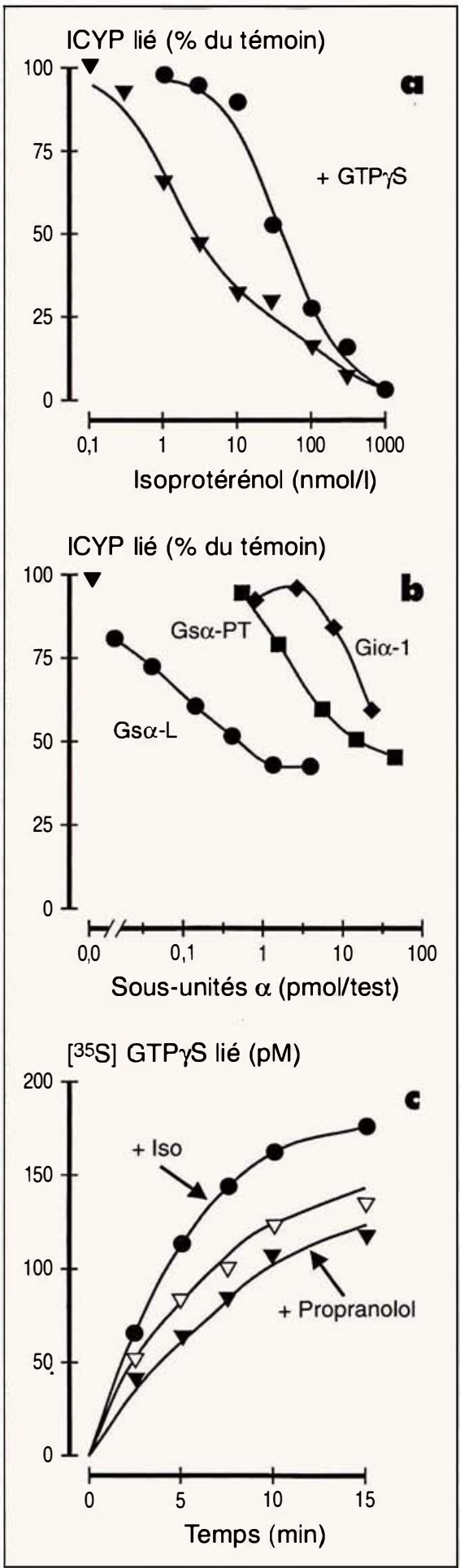
teur est retrouvée, comme en témoigne l'augmentation de la vitesse de liaison $[\mathrm{GTP} \gamma-\mathrm{S}]$ radiomarqué dans les membranes en présence d'isoprotérénol.

L'ensemble de ces expériences a montré que, pour le système $\beta$ adrénergique, l'expression dans $E$ coli permet de reconstituer un couplage fonctionnel bidirectionnel entre récepteur et protéine $\mathrm{G}$.

\section{Couplage fonctionnel entre récepteurs 5-HT1-A et protéines $G$ exprimés dans E.coli}

Pour poursuivre l'évaluation du système de reconstitution décrit plus haut, nous avons choisi d'étudier le couplage du récepteur sérotoninergique 5HT1-A [7]. Ce choix a été motivé par plusieurs raisons : (1) la séquence de ce récepteur est relativement homologue à celle du récepteur $\beta 2$ adrénergique (le gène a d'ailleurs été cloné en utilisant une sonde $\beta 2$ ), ce qui nous faisait espérer une expression équivalente dans les bactéries ; (2) ce récepteur se couple à des protéines $\mathrm{Gi}$, ce qui n'est pas le cas du récepteur $\beta 2$; (3) certaines expériences suggéraient que ce récepteur pourrait soit activer, soit inhiber l'adénylate cyclase ; cette étude devait permettre de vérifier si le récepteur 5HT1-A pouvait effectivement se coupler avec des protéines $\mathrm{Gs}$ et $\mathrm{Gi}$. Suivant le procédé expérimental utilisé dans le cas des récepteurs $\beta$ adrénergiques, le couplage fonctionnel du récepteur $5 \mathrm{HT} 1-\mathrm{A}$ a été étudié en présence de $\mathrm{rGI} \alpha-1, \mathrm{rGI} \alpha-2$, $\mathrm{rGI} \alpha-3$, rGo $\alpha, \mathrm{rGs} \alpha$ et $\mathrm{rGs} \alpha-\mathrm{L}$. L'ordre d'affinité apparente du récepteur pour les protéines $G$ s'établit comme suit : $\mathrm{rGi} \alpha-3>\mathrm{rGi} \alpha-2>$ $\mathrm{rGi} \alpha-1>\mathrm{RGo}$ (figure 4). Ni $\mathrm{rGs} \alpha$-S ni $\mathrm{rGs} \alpha-\mathrm{L}$ ne semblent capables d'interagir avec le récepteur 5H1-TA. La comparaison de l'affinité apparente de couplage pour rGI $\alpha$ par rapport à celle pour une préparation de $\mathrm{Gi}$ et Go à partir de cerveau bovin suggère que les protéines recombinantes exprimées dans $E$. coli sont environ 20 fois moins efficaces que celles purifiées à partir de tissus de mammifère. Cette plus faible affinité pourrait être due à l'absence de miristylation des sous-unités $\mathrm{Gi}$ et Go dans
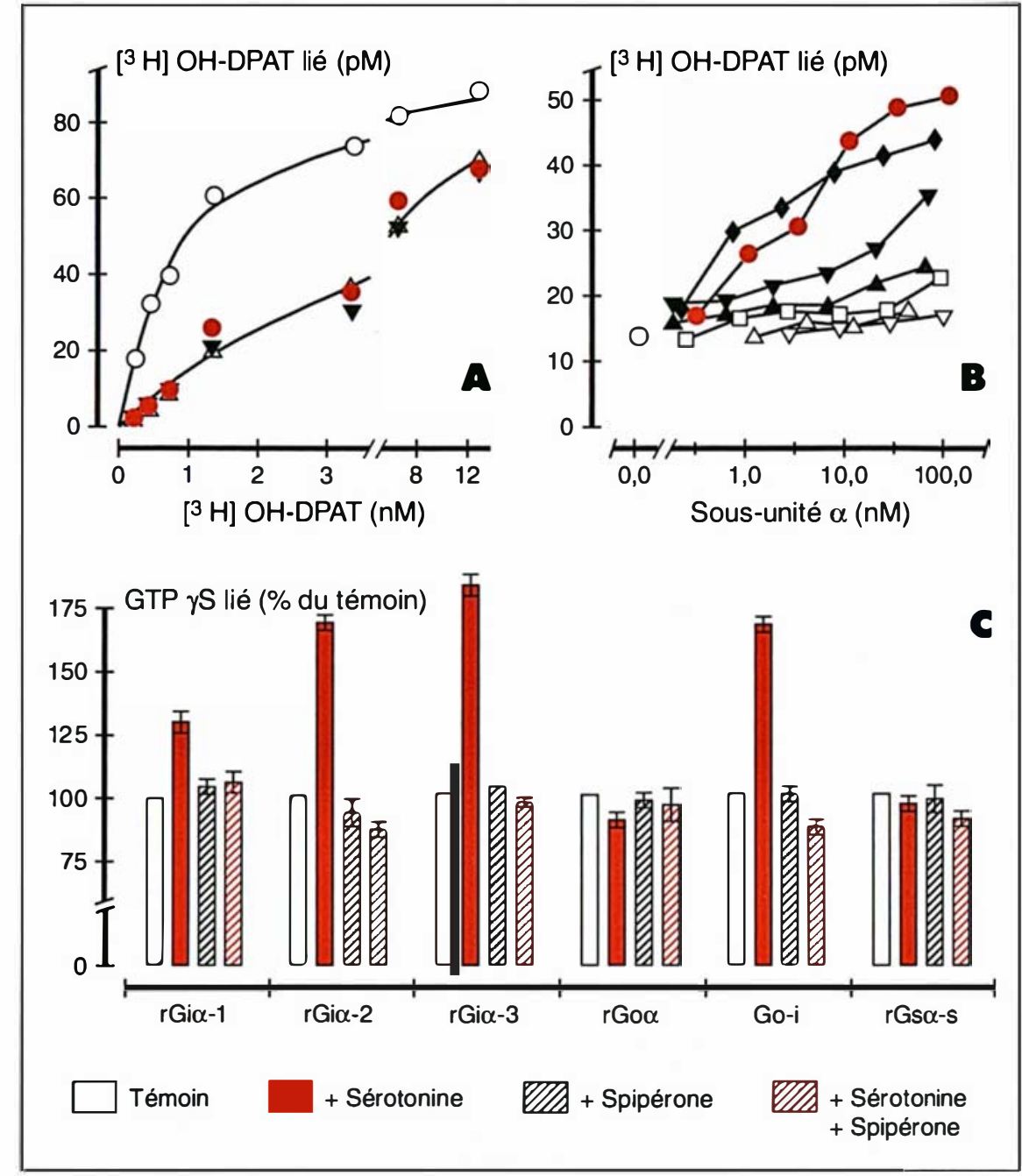

Figure 4. Mise en évidence du couplage entre récepteurs 5-HT1A de la sérotonine et sous-unités $\alpha$ recombinantes des protéines $\mathbf{G}$ exprimés dans E. coli. Le procédé de reconstitution est identique à celui décrit dans la figure 3. (A) Expériences de saturation du récepteur 5-HT1A recombinant par un ligand agoniste (le $\left.{ }^{[3} \mathrm{H}\right]$ 8-OH-DPAT) en présence $(\mathrm{O})$ ou en absence $(\Delta \nabla)$

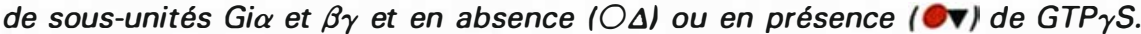

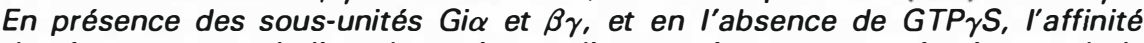
du récepteur pour le ligand agoniste radiomarqué augmente, témoignant de la reconstitution du complexe ternaire. (B) Titration du récepteur 5-HT1A en présence de concentrations croissantes de sous-unités $G \alpha$ recombinantes. Dans le milieu réactionnel, les récepteurs sont en présence de concentrations fixes de ligand agoniste radiomarqué (le $\left[{ }^{3} \mathrm{H}\right]$ 8-OH-DPAT). Plus on ajoute de sousunité $G \alpha$, plus l'agoniste est lié si le couplage est reconstitué. Cette figure montre que l'ordre préférentiel de couplage du récepteur 5-HT1A est Gio-3 $(\nabla)>\operatorname{Gi\alpha }-2(\nabla)>\operatorname{Gi\alpha }-1(\Delta)>G o \alpha(\square)$. Deux variantes d'épissage de Gs $\alpha$ $(\Delta \nabla)$ ne se couplent pas au récepteur même à des concentrations de $100 \mathrm{nM}$. La préparation de protéines Go-i (O) directement purifiées du cerveau bovin permet le meilleur couplage. Ce fait est vraisemblablement dû à l'absence de myristylation des sous-unités a recombinantes. (C) Incorporation de GTP $\gamma S$ radiomarqué par les différentes sous-unités $\alpha$ recombinantes, ou purifiées à partir du cerveau bovin (Go-i), en présence de membranes bactériennes exprimant des récepteurs 5-HT1A et des ligands indiqués. La sérotonine stimule l'incorporation et cet effet est inhibé par un excès de ligand antagoniste (spipérone). Le même ordre de couplage que celui mis en évidence par l'expérience précédente est retrouvé. 
$E$. coli. Cependant, on ne peut pas exclure la présence dans la préparation de $\mathrm{Gi}$ et Go bovines d'une protéine $G$ non encore identifiée et qui interagirait avec une haute affinité avec le récepteur $5 \mathrm{HT}-1 \mathrm{~A}$.

\section{Conclusions}

L'expression des récepteurs dans $E$. coli est maintenant utilisée pour de multiples applications. C'est devenu un outil éprouvé pour le criblage de ligands, comme en témoigne son exploitation au niveau industriel [17, 18]. Nous avons utilisé cet outil pour exprimer des récepteurs $\beta$ adrénergiques mutants destinés à l'étude du site de liaison des ligands $[19,20]$, et il pourrait ainsi être adopté par ceux qui s'intéressent à la modélisation du site de liaison des récepteurs couplés aux protéines G.

Nous savons maintenant qu'il peut également être employé comme un système de reconstitution sans "bruit de fond " pour analyser des mécanismes complexes d'interaction entre macromolécules, comme par exemple les bases structurales du couplage entre récepteurs et protéines G. Dans cette application, ce système a des inconvénients surtout liés aux différences de transformation que les protéines néo-synthétisées subissent dans les bactéries par rapport aux cellules des organismes supérieurs. Nous sommes cependant convaincus qu'il s'agit d'un système perfectible et que les bactéries nous permettrons d'apprendre encore beaucoup sur le fonctionnement des récepteurs humains

\section{Remerciements}

Les travaux résumés ici sont le fruit de nombreuses discussions et collaborations avec des collègues du laboratoire, notamment Laurent Emorine et Jean-Gérard Guillet, ou d'autres laboratoires, en particulier celui de Maurice Hofnung à l'Institut Pasteur. Nos recherches sont soutenues par le Cnrs, l'Inserm, l'université Paris-VII et le MRE.

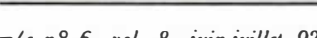

\section{Summary}

When bacteria show how human receptors work

$\mathrm{G}$ protein-coupled membrane receptors are involved in the homeostatic regulation of many central functions of the body. They activate heterotrimeric $G$ proteins which are comprised of $\alpha$, $\beta$ and $\gamma$ subunits. The $\alpha$ subunits couple with receptors and activate in turn cellular effectors such as enzymes or ion channels. More than 85 receptors and $20 \alpha$ subunits, which are involved in the complex network of cellular signaling, have already been identified. The molecular basis of coupling selectivity between receptors and $\mathrm{G}$ proteins is poorly understood and difficult to study, due to the co-expression of multiple receptors and $G$ proteins in the cells of higher organisms. $G$ protein-coupled receptors expressed in Escherichia coli have been shown to maintain their ligand binding properties. Similarly, several G-protein $\alpha$ subunits can be expressed in bacteria and may interact with receptors after purification. These advances are the basis of an in vitro procedure designed to reconstitute the coupling between receptor and $\mathrm{G}$ proteins both expressed in Escherichia coli. Coupling preferences between a single receptor and several $G$ proteins may be evaluated with this approach, which avoids any background due to the presence of endogeneous receptors or $\mathrm{G}$ proteins.

\section{TIRÉS A PART}

